\title{
Série histórica de mortalidade por neoplasias renais no Brasil (1996-2010)
} Time series of renal cancer mortality in Brazil (1996-2010)

\author{
Genesis de Souza Barbosa1', Roberta de Lima², Raphael Mendonça Guimarães ${ }^{3}$
}

\begin{abstract}
Resumo
O presente artigo tem como objetivo avaliar a tendência da mortalidade por neoplasia renal no Brasil entre 1996 e 2010 . Foi realizado um estudo de série temporal cuja taxa foi padronizada pela população mundial e log-transformada. Em seguida, houve regressão polinomial para verificar a tendência das taxas, que revela maior suscetibilidade nos idosos, seguidos pelo grupo dos adultos e o de crianças de zero a nove anos. Nesta lógica, ações de caráter preventivo, sobretudo direcionadas às populações mais vulneráveis, podem trazer benefícios, diminuindo o número de óbitos por neoplasias renais no Brasil.

Palavras-chave: neoplasias renais; neoplasias; epidemiologia.
\end{abstract}

\begin{abstract}
This article aims to evaluate the trend of renal cancer mortality in Brazil between 1996 and 2010. It was studied a time series which the rate was standardized by world population and log-transformed. Then, polynomial regression was performed to verify the rates trend that shows higher susceptibility in the elderly, followed by the group of adults and children with zero to nine years old. With this logic, preventive actions, especially targeted to vulnerable populations, may bring benefits, minimizing the number of renal cancer deaths in Brazil.
\end{abstract}

Keywords: kidney neoplasms; neoplasms; epidemiology.

Trabalho realizado na Universidade Federal do Rio de Janeiro (UFRJ) - Rio de Janeiro (RJ), Brasil.

${ }^{1}$ Mestre em Enfermagem pela Escola de Enfermagem Anna Nery da UFRJ; Professor Substituto do Curso de Enfermagem e Obstetrícia da UFRJ - Macaé (RJ), Brasil.

2Mestre em Enfermagem pela Escola de Enfermagem Anna Nery da UFRJ; Professora Assistente do Curso de Enfermagem e Obstetrícia da UFRJ - Macaé (RJ), Brasil.

${ }^{3}$ Doutor em Saúde Coletiva pelo Instituto de Estudos em Saúde Coletiva da UFRJ; Professor Adjunto do Instituto de Estudos em Saúde Coletiva da UFRJ - Rio de Janeiro (RJ), Brasil.

Endereço para correspondência: Genesis de Souza Barbosa - Rua Aluísio da Silva Gomes, 50 - Granja dos Cavaleiros - CEP: 27930-560 - Macaé (RJ), Brasil E-mail: genesisbarbosa@gmail.com.

Fonte de financiamento: nenhuma.

Conflito de interesse: nada a declarar. 


\section{INTRODUÇÃO}

A neoplasia renal é uma doença heterogênea, constituída por um número de subtipos histológicos associados a mutações genéticas, diferentes características clínicas e sensibilidade ao tratamento, sendo o carcinoma de células renais claras o subtipo histológico mais comum ${ }^{1-3}$. Sua incidência é crescente a partir de 1970 e, em parte, essa progressão é atribuída ao advento de inovações no campo da imagem, permitindo a detecção de tumores não localizados por métodos empregados anteriormente 4 .

Nos Estados Unidos, as neoplasias renais estão entre os dez tipos mais comuns da doença ${ }^{3}$. Entretanto, no Brasil, não figuram entre os 15 tipos mais comuns de câncer e há, atualmente, uma lacuna no que diz respeito aos dados isolados desta patologia, englobada nas estatísticas gerais dos subgrupos menos incidentes 5 . No cenário atual, os dados sobre mortalidade por neoplasias renais nos permitem conhecer o universo de pessoas que morrem por alguma das condições malignas de tumor renal. Assim, este estudo pretende avaliar a tendência da mortalidade por neoplasia renal no Brasil entre 1996 a 2010.

\section{MÉTODOS}

Foi realizado um estudo de série temporal, baseado em dados secundários coletados do Departamento de Informática do Sistema Único de Saúde (DATASUS). Para as análises foram incluídos os códigos de Classificação Estatística Internacional de Doenças e Problemas Relacionados à Saúde (CID10) pertinentes à temática, a saber: CID-C64 Neoplasia maligna do rim, exceto pelve renal; e CID-C65 Neoplasia maligna da pelve renal.

As taxas de mortalidade específicas e ajustadas por idade por 100 mil pessoas foram calculadas para cada ano, entre $1996 \mathrm{e}$ 2010, e também para as faixas etárias, utilizando intervalos de dez anos. Elas foram ajustadas com base na população padrão mundial para comparações globais ${ }^{6}$.

Na sequência foi iniciado o processo de modelagem, considerando as taxas de mortalidade como variável dependente $(\mathrm{Y})$ e os anos de estudo como variável independente (X). Para o estudo da tendência, optou-se por estimar modelos de regressão, e a fim de evitar a colinearidade entre os termos da equação de regressão, utilizou-se a variável centralizada. $\mathrm{O}$ primeiro modelo a ser testado foi o de regressão linear simples $\left(\mathrm{Y}=\beta_{0}+\beta_{1} \mathrm{X}\right)$ e, posteriormente, foram testados os modelos de ordem maior: segundo grau ou parabólico $\left(Y=\beta_{0}+\beta_{1} X+\beta_{2} X^{2}\right)$ e terceiro grau $\left(Y=\beta_{0}+\beta_{1} X+\beta_{2} X^{2}+\beta_{3} X^{3}\right)$. Considerou-se o melhor modelo aquele que apresentou maior coeficiente de determinação $\left(\mathrm{R}^{2}\right)^{7}$. Quando dois modelos foram semelhantes para a mesma localidade, do ponto de vista estatístico, optou-se pelo mais simples, ou seja, de menor ordem.

\section{RESULTADOS E DISCUSSÃO}

As Figuras 1 e 2 apresentam, respectivamente, a tendência da taxa de mortalidade por neoplasias renais no Brasil por faixa etária e a distribuição da taxa de mortalidade por

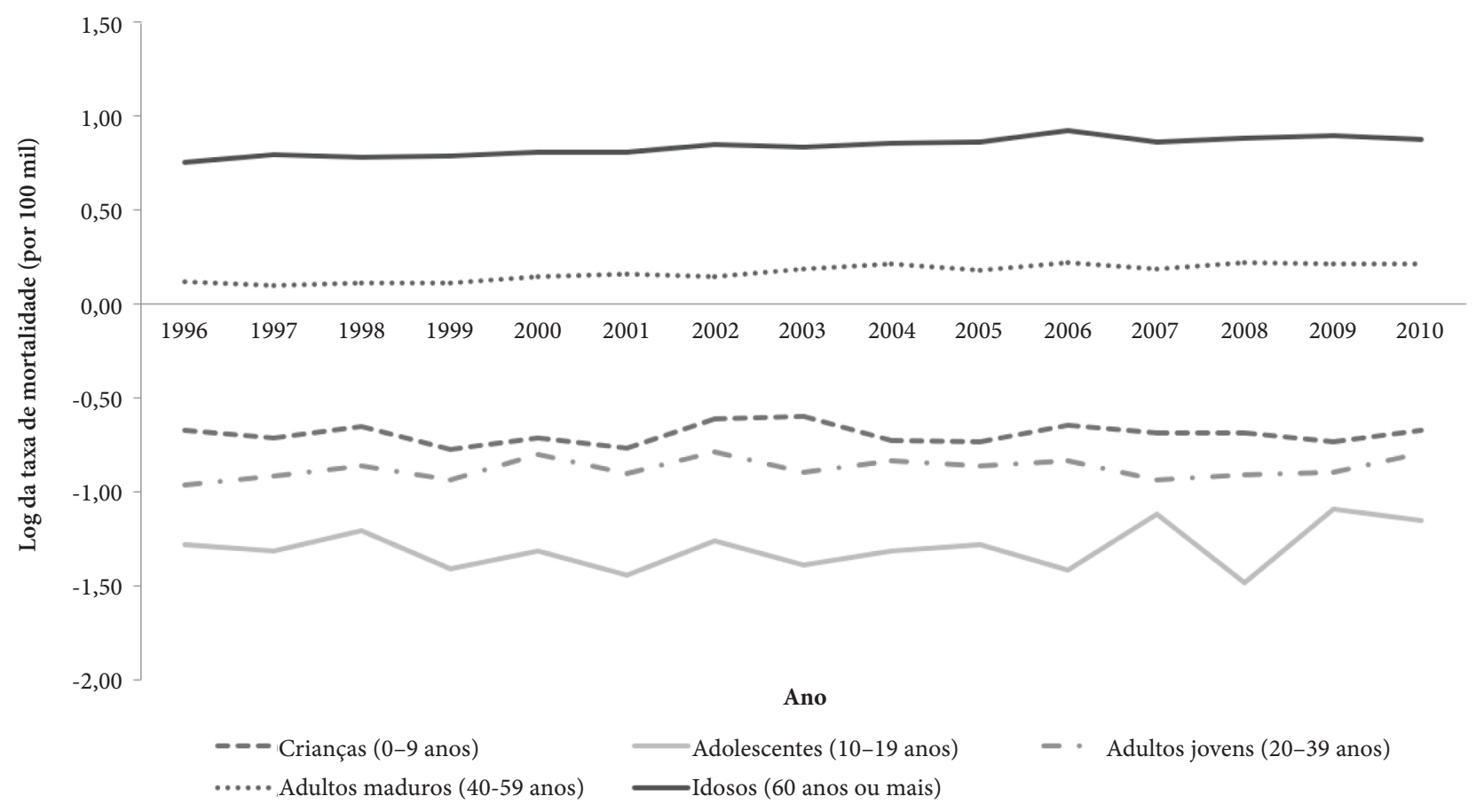

Figura 1. Tendência da taxa de mortalidade por neoplasias renais no Brasil por faixa etária entre 1996 e 2010 


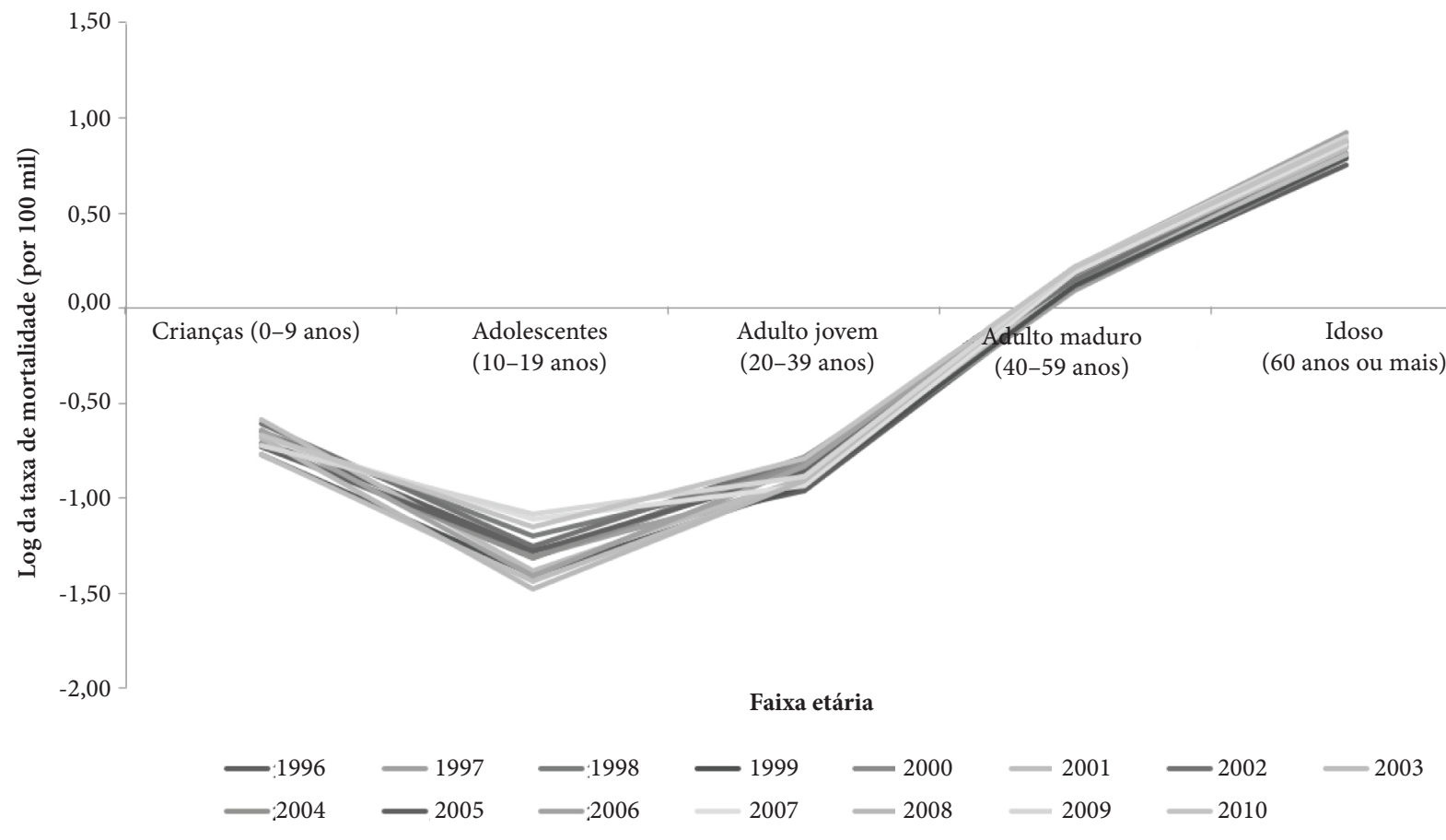

Figura 2. Distribuição da taxa de mortalidade por neoplasias renais no Brasil segundo a faixa etária, entre 1996 e 2010

neoplasias renais no Brasil segundo a faixa etária. A primeira revela maior suscetibilidade nos idosos, o que corrobora com a tendência mundial ${ }^{3}$, seguidos pelo grupo dos adultos maduros no qual, em geral, se conhece o diagnóstico ${ }^{2-4,8}$, e o de crianças de zero a nove anos de idade, ocupando o terceiro lugar, com maior variabilidade da taxa de mortalidade ao longo dos anos.

Sobre a distribuição, observa-se o efeito da idade nas taxas, obedecendo a um padrão constante ao longo dos anos. A taxa de mortalidade por neoplasias renais no Brasil no período estudado reafirma a tendência global em que a maioria dos indivíduos acometidos é idosa ${ }^{3,8-11}$. A idade média de diagnóstico é 64 anos, sendo muito raro o diagnóstico em indivíduos com menos de 45 anos e ocorrendo com maior frequência em pessoas com 55 anos $^{3}$. Ainda, as chances de desenvolver algum tipo de câncer renal dobram em pessoas com diabetes ou hipertensão arterial, doenças tipicamente diagnosticadas e agravadas em idades mais remotas, o que justifica a ocorrência deste câncer em fase mais avançada da vida ${ }^{12}$.

\section{CONCLUSÃO}

As neoplasias renais no Brasil são responsáveis por mais mortes de idosos e têm maior incidência na faixa compreendida entre 40 e 60 anos de idade. Crianças e adolescentes representam o menor grupo afetado pelas mortes causadas por neoplasias renais. Doenças crônicas como diabetes e hipertensão representam fatores de risco para o surgimento da doença.

Posto isto, discutir a mortalidade por neoplasia no Brasil constitui uma importante ferramenta, uma vez que aponta para um problema de saúde pública. Nesta lógica, ações de caráter preventivo, sobretudo direcionadas às populações mais vulneráveis, podem trazer benefícios, diminuindo o número de óbitos por neoplasias renais no Brasil.

\section{REFERÊNCIAS}

1. Singer EA, Bratslavsky G, Middelton L, Srinivasan R, Linehan WM. Impact of genetics on the diagnosis and treatment of renal cancer. Curr Urol Rep. 2011;12(1):47-55.

2. Koul H, Huh JS, Rove KO, Crompton L, Koul S, Meacham RB, Kim FJ. Molecular aspects of renal cell carcinoma: a review. Am J Cancer Res. 2011;1(2):240-54.

3. American Cancer Society. Cancer facts and figures 2012. American Cancer Society [Internet]. [cited 2012 Apr 09]. Available from: http:// www.cancer.org/research/cancerfactsfigures/cancerfactsfigures/cancerfacts-figures-2012

4. Maciel LC, Nascimento LF, Elias CPF, Rodrigues BS, Oliveira FLG, Bertti ROT. A ultra-sonografia como método de rastreamento populacional de tumor renal. Rev Col Bras Cir. 2007;34(6):398-400.

5. Ministério da Saúde. Instituto Nacional de Câncer José Alencar Gomes da Silva. Estimativa 2012: incidência de câncer no Brasil. Rio de Janeiro: INCA, 2011. 
6. Doll R, Waterhouse J, Payne P. Cancer incidence in five continents: a technical report. Genéve: International Union against Cancer; 1966.

7. Latorre MRDO, Cardoso MRA. Análise de séries temporais em epidemiologia: uma introdução sobre os aspectos metodológicos. Rev Bras Epidemiol. 2001;4(3):145-52.

8. Paula TA, Silva PSL, Berriel LGS. Carcinoma de células renais com metástase cutânea: relato de caso. J Bras Nefrol. 2010;32(2):213-5.

9. Bhat S. Role of surgery in advanced/metastatic renal cell carcinoma. Indian J Urol. 2010;26(2):167-76.
10. Dall'Oglio M, Srougi M, Ortiz V, Nesrallah L, Gonçalves PD, Leite KM, Hering F. Carcinoma de células renais incidentais e sintomáticos: fatores patológicos e sobrevida. Rev Assoc Med Bras. 2004;50(1):27-31.

11. Instituto Brasileiro de Geografia e Estatística (IBGE). Pesquisa Nacional por Amostra de Domicílios 2008. Rio de Janeiro: IBGE, 2008.

12. Habib SL, Prihoda TJ, Luna M, Werner SA. Diabetes and risk of renal cell carcinoma. J Cancer. 2012;3:42-8.

Recebido em: 20/04/2012 Aprovado em: 13/09/2012 